Check for updates

Cite this: Mater. Adv., 2022, 3, 1759

Received 6th December 2021, Accepted 30th December 2021

DOI: 10.1039/d1ma01144h

rsc.li/materials-advances

\title{
Stable dinitrile end-capped closed-shell non-quinodimethane as a donor, an acceptor and an additive for organic solar cells $\dagger$
}

\author{
Weixuan Liang, $\ddagger^{a}$ Peng Liu,,$^{a}$ Yiheng Zhang, ${ }^{a}$ Weiya Zhu, ${ }^{a}$ Xinyang Tao, ${ }^{a}$ \\ Zhicai He (D)*a and Yuan Li (iD)*ab
}

\begin{abstract}
Non-fullerene acceptors exhibit great potential in improving the photovoltaic performances of organic solar cells. However, it is important to further enhance their chemical stability and device durability for future commercialization, especially for Y6-series small molecule acceptors with 2-(3-oxo-2,3dihydroinden-1-ylidene)malononitrile (IC) as the ending group. In this work, an IC-free photovoltaic material YF-CN consisting of the 2-fluoren-9-ylidenepropanedinitrile terminal was designed and synthesized by Stille coupling. YF-CN has a closed-shell chemical structure with enhanced photostability and improved morphological compatibility with the binary PCE10:Y6 blend. The moderate energy level enables YF-CN to serve as a multifunctional material, such as a donor, an acceptor and a third component. When adding YF-CN as a second donor into the PCE10:Y6 system, an improved power conversion efficiency of $12.03 \%$ was achieved for the as-cast device. Importantly, the ternary PCE10:YFCN:Y6-devices showed enhanced storage durability maintaining 91\% of their initial PCE after storing for $360 \mathrm{~h}$. This work provides a new perspective to understand the open-shell character of the donor and the closed-shell structure of the acceptor, as well as a promising design concept of stable IC-free acceptors for organic solar cells.
\end{abstract}

\section{Introduction}

Organic solar cells (OSCs) are considered to be the next potentially commercialized photovoltaic technology owing to their advantages including light weight, flexibility and largescale fabrication via solution processing. ${ }^{1-5}$ The active layer of conventional OSCs usually comprises a binary blend bulk heterojunction (BHJ) of the electron donor and acceptor, which plays a critical role in determining the performance of the devices. ${ }^{6,7}$ In the last 10 years, as immense efforts have been dedicated to the molecular design of non-fullerene acceptors (NFAs), the power conversion efficiencies (PCEs) of singlejunction OSCs have made great progress. ${ }^{8-18}$

Different from binary OSCs, ternary OSCs appear to be a viable tool to extend light absorption, facilitate exciton dissociation and optimize the morphology, leading to an improvement of

\footnotetext{
${ }^{a}$ Institute of Polymer Optoelectronic Materials and Devices, State Key Laboratory of Luminescent Materials and Devices, South China University of Technology,

Guangzhou, 510640, P. R. China. E-mail: celiy@scut.edu.cn, zhicaihe@scut.edu.cn ${ }^{b}$ Guangdong Provincial Key Laboratory of Luminescence from Molecular Aggregates, South China University of Technology, Guangzhou, 510640, P. R. China

$\dagger$ Electronic supplementary information (ESI) available. See DOI: 10.1039/ d1ma01144h

\$ These authors contributed equally to this work.
}

photovoltaic performances. ${ }^{19-27}$ Cascade energy alignment could be achieved by introducing a third component with suitable energy levels, which is beneficial to suppress charge recombination. ${ }^{28,29}$ Another significant function of the third component is to improve the compatibility and miscibility with the binary blend films. ${ }^{30-34}$ However, it should be noted that the addition of a third component also might result in a more complex morphology and create difficulties in the morphology control of some ternary systems. ${ }^{35}$ By selecting an appropriate third component, PCEs more than $18 \%$ have been reported for efficient ternary OSCs, which indicates tremendous potential for development. ${ }^{36-42}$

In addition to high efficiency, device stability is also an important factor for solar cells to be commercialized. ${ }^{43-45}$ For Y6-series non-fullerene solar cells, the key to achieve high device stability is to stabilize the microstructure of blend films. ${ }^{46}$ Furthermore, most emerging NFAs containing 2-(3oxo-2,3-dihydroinden-1-ylidene)malononitrile (IC)-type terminals are synthesized by the Knoevenagel condensation reaction, which would make them prone to decompose under persistent light irritation. ${ }^{47-53}$ It has been demonstrated that the photodegradation products of typical NFAs such as ITIC with IC end groups contain fused-ring isomers via a 6-e electrocyclic reaction between the dicyanomethylene unit and 

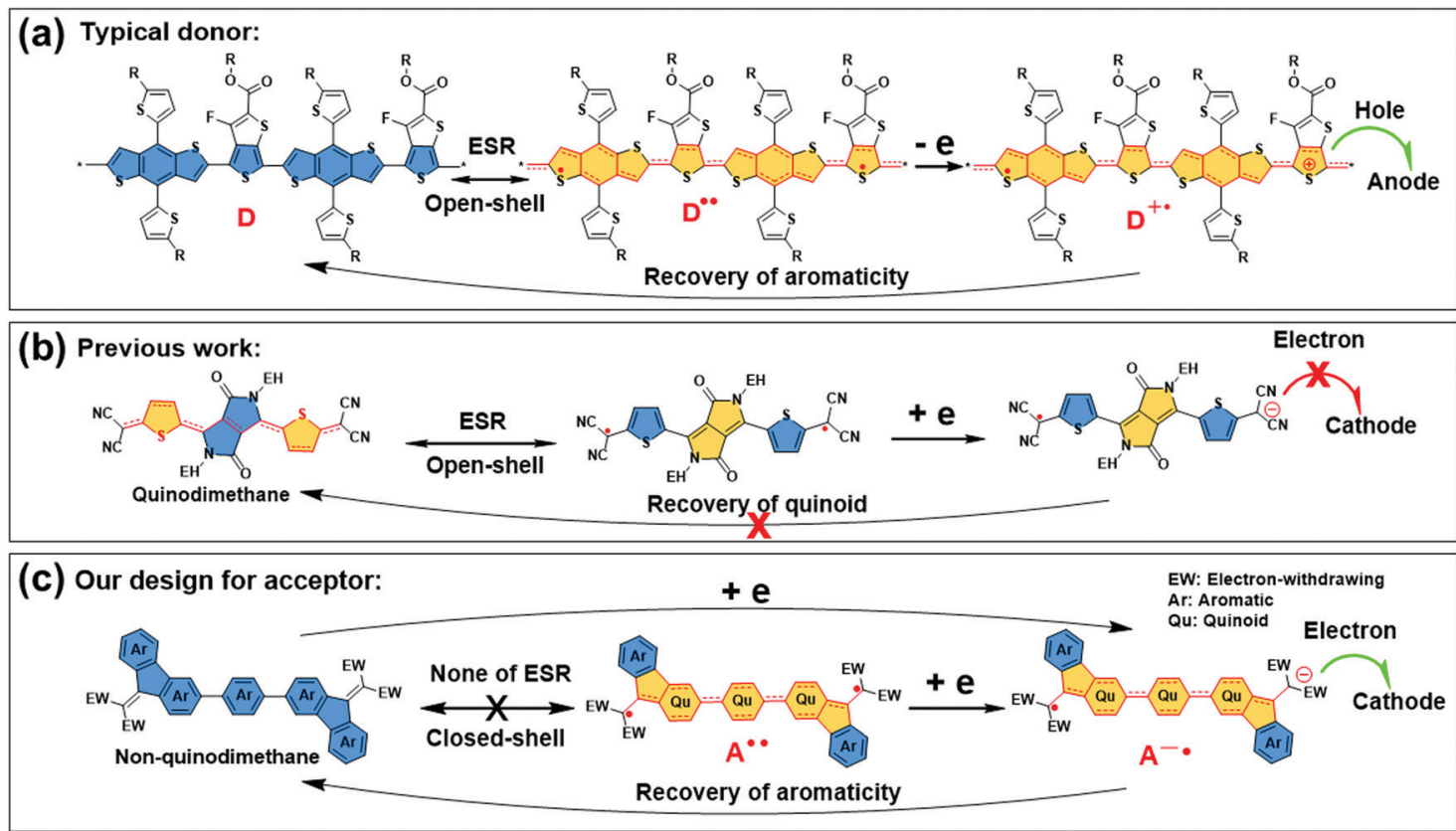

Scheme 1 (a) The resonance structure of a typical open-shell donor PCE10 and the formation of radical cation during the operation of OCSs. (b) Previous resonance structure of open-shell CN-TDPP based on 2,5-bis(2-ethylhexyl)-3,6-di(thiophen-2-yl)-2,5-dihydropyrrolo-[3,4-c]pyrrole-1,4dione (TDPP) and the formation of radical anion during the operation of OCSs. (c) Our design on closed-shell acceptor and the formation of radical anion during the operation of OCSs.

the thiophene ring. ${ }^{50} \mathrm{~A}$ promising and efficient method is employing robust carbon-carbon bonds instead of the vulnerable exocyclic double bonds to link the donor unit and the acceptor unit of NFAs, which can not only lead to intrinsically stable acceptors but also successfully adjust the optoelectronic properties of NFAs. ${ }^{53}$ The development of IC-free NFAs is still very challenging, ${ }^{54}$ to date.

In our previous work, we have disclosed the open-shell radical character of donor-acceptor (D-A) type organic semiconductors. ${ }^{55-58}$ As presented in Scheme 1, the donors of OSCs including PCE10 and small molecules show a quinoidal radical resonance structure which will produce a radical cation and recover the aromatic neutral form during the operation of OSCs (Scheme 1a). ${ }^{59-62}$ In previous studies, $p$-quinodimethane CN-TDPP was reported to be a typical diradicaloid with an open-shell singlet ground state and showed poor performance in OSCs as the radical anion will be stabilized while it formed the aromatic resonance structure (Scheme 1b). ${ }^{56,57}$ Based on the above considerations, we proposed a new strategy to construct a closed-shell aromatic (non-quinodimethane) backbone with dinitrile end-capping groups for the design of NFAs in OSCs (Scheme 1c), and this will provide the expected high stability than IC-based NFAs. It is noteworthy that the radical anion will more readily recover into its aromatic neutral form compared to p-quinodimethane in scheme $1 \mathrm{~b}$, due to the well-known aromatic stabilization energy effect detected in previous Chichibabin type diradicaloids. ${ }^{56}$ Interestingly, we detected that the donors show an open-shell structure, and the efficient NFAs including ITIC and Y6-series have closedshell structures as these NFAs exhibit no or extremely weak ESR signal, while the poor acceptors including CN-TDPP and others have open-shell structure as they exhibit a strong ESR signal and bipolar transport properties. ${ }^{5-58}$

Herein, we reported an IC-free organic small molecule named YF-CN with facile synthesis steps by changing the terminal group of $\mathrm{Y} 6$ into 2-fluoren-9-ylidenepropanedinitrile. Compared with Y6, YF-CN shows good photostability and higher energy levels. The achievement of PCEs for binary OSCs based on PCE10:YF-CN and YF-CN:Y6 blends suggests that YF-CN exhibits unprecedented and unique bipolar charge accepting capability, that is, it can either work as an electron acceptor or as an electron donor, in non-fullerene organic solar cells. This also makes it efficient as the third component to improve the ternary OSC device efficiency of the classic material system of PCE10:Y6, resulting in an elevated PCE of $12.03 \%$ without solvent addition and posttreatment. The improved PCE of the ternary OSCs originates from the increased $V_{\mathrm{OC}}$ and $J_{\mathrm{SC}}$ compared with that of PCE10:Y6-based OSCs. This is supposed to increase as a consequence of the enhanced exciton separation, optimized morphology and improved charge transport mobility facilitated by the addition of the third component. Furthermore, better storage stability was realized in ternary devices whose PCE showed a slight decrease of merely $9 \%$ after storing for $360 \mathrm{~h}$ because of the enhanced microstructure stability of the ternary active layer.

\section{Results and discussion}

The synthesis of YF-CN consists of two steps, which are described in the ESI. $\uparrow$ Nuclear magnetic resonance (NMR) 

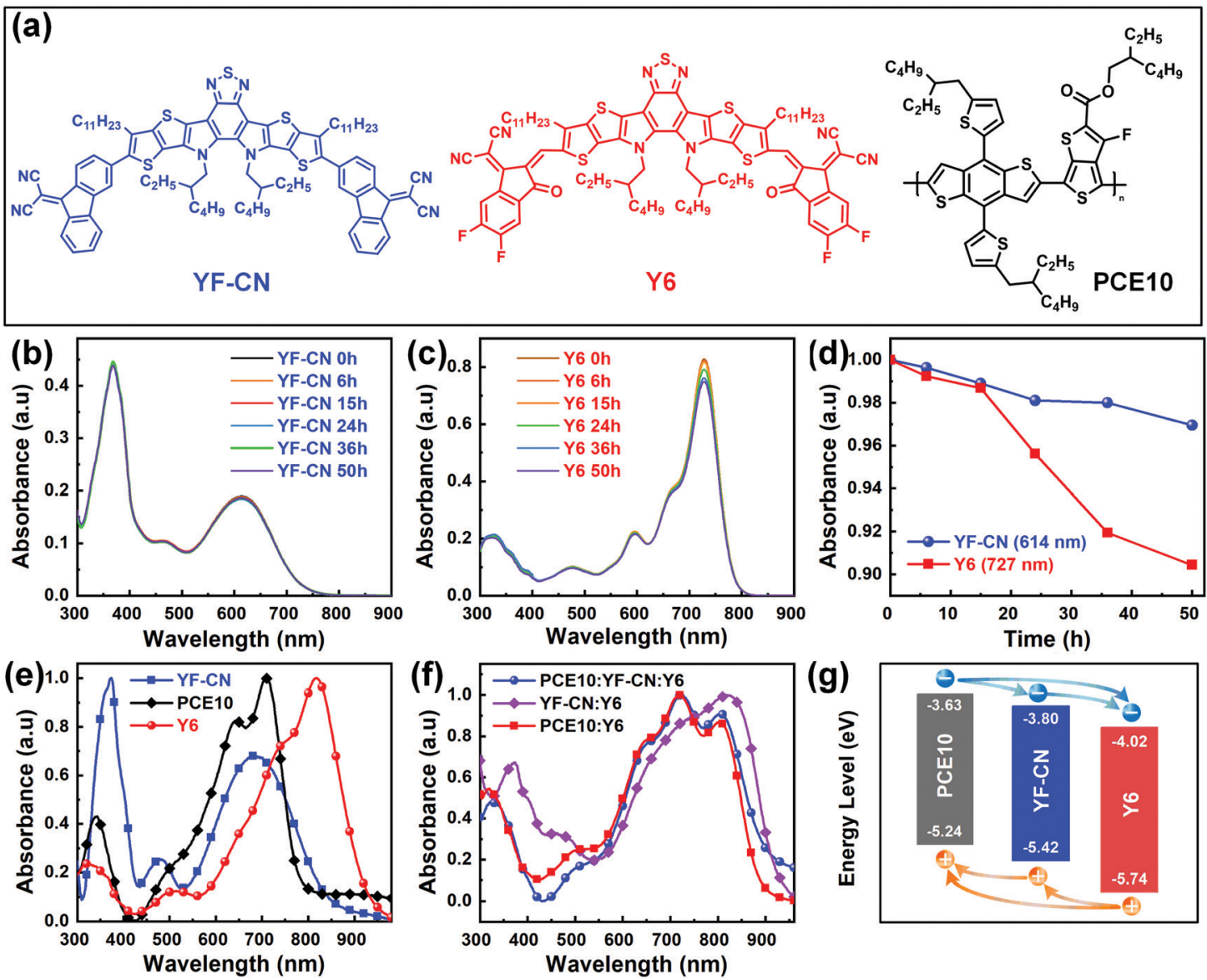

Fig. 1 (a) Molecular structure of the acceptor YF-CN, PCE10 and Y6. Solution aging time-dependent UV-vis absorption of (b) YF-CN and (c) Y6 upon irradiation using a $100 \mathrm{~W}$ tungsten lamp in chlorobenzene solution. (d) The change of absorption intensity of YF-CN and Y6 under different aging times. (e) UV-vis absorption spectra of films of above materials. (f) UV-vis absorption spectra of blend films spin-coated via toluene solution. (g) Energy levels of PCE10, YF-CN and Y6 and the paths of charge transfer.

spectra and the high-resolution matrix-assisted laser desorption/ ionization time of flight (MALDI-TOF) mass spectrum of the intermediate and YF-CN are shown in Fig. S1-S5 (ESI †). Fig. 1a presents the chemical structures of active materials PCE10, YF$\mathrm{CN}$ and Y6. The optical and electrochemical parameters of PCE10, YF-CN and Y6 are summarized in Table 1. Compared with the solution, the YF-CN film exhibited an absorption peak at $687 \mathrm{~nm}$ that is red-shifted by $\sim 70 \mathrm{~nm}$ (Fig. S6, ESI $\dagger$ ), indicating the existence of strong $\pi-\pi$ interactions and charge transfer in the solid film. ${ }^{63}$ The YF-CN film has broad absorption in the range of 500-800 $\mathrm{nm}$ with the absorption onset located at $838 \mathrm{~nm}$, leading to an optical bandgap of $1.48 \mathrm{eV}$. To assess the

Table 1 The optical and electrochemical parameters of PCE10, YF-CN and $Y 6$

\begin{tabular}{llllll}
\hline Materials & $\lambda_{\text {peak }}^{\text {film }}(\mathrm{nm})$ & $\lambda_{\text {onset }}^{\text {film }}(\mathrm{nm})$ & $E_{\mathrm{g}}^{\text {opt }}(\mathrm{eV})^{a}$ & HOMO $(\mathrm{eV})^{b}$ & LUMO $(\mathrm{eV})^{c}$ \\
\hline PCE10 & 707 & 772 & 1.61 & -5.24 & -3.63 \\
YF-CN & 370,687 & 838 & 1.48 & -5.42 & -3.80 \\
Y6 & 816 & 932 & 1.33 & -5.74 & -4.02
\end{tabular}

${ }^{a} E_{\mathrm{g}}^{\mathrm{opt}}=1240 / \lambda_{\mathrm{onset}}^{\mathrm{film}}(\mathrm{eV}) \cdot{ }^{b} \mathrm{HOMO}=-\left(E_{\mathrm{ox}}+4.80-E_{\mathrm{f}}\right)(\mathrm{eV}) \cdot{ }^{c} \mathrm{LUMO}=$ $-\left(E_{\text {red }}+4.80-E_{\mathrm{f}}\right)(\mathrm{eV})$. photostability of YF-CN and Y6, we conducted their absorption decay test in chlorobenzene solutions under illumination of a $100 \mathrm{~W}$ tungsten lamp. As displayed in Fig. 1b-d, YF-CN exhibited high photostability with almost constant absorption intensity, while a significant attenuation was found in the absorption of Y6. The improved photostability can be attributed to the advantages of having robust $\mathrm{C}-\mathrm{C}$ single bonds, rather than delicate exocyclic $\mathrm{C}=\mathrm{C}$ bonds. ${ }^{53}$ As for the absorption of films, when compared with Y6, the absorption peak of YF-CN exhibits an apparent blue-shift (Fig. 1e) because of the weaker electron withdrawing ability of the ending group, which leads to an attenuated intramolecular charge transfer interaction. ${ }^{64}$ In addition, the absorption of YF-CN exhibits a partial overlap with the absorption of PCE10. Therefore, compared with the absorption position of PCE10:Y6, the introduction of the third component YFCN partially replacing PCE10 does not change the light absorption obviously in the ternary PCE10:YF-CN:Y6 blend films (Fig. 1f).

The highest occupied molecular orbital (HOMO) energy levels and the lowest unoccupied molecular orbital (LUMO) energy levels of materials were measured by the cyclic voltammetry (CV) method (Fig. S7, ESI†). Fig. 1g shows the corresponding energy levels of the three photovoltaic materials 
and the paths of charge transfer. The LUMO and HOMO levels of YF-CN were determined to be $-3.80 \mathrm{eV}$ and $-5.42 \mathrm{eV}$, respectively. A cascaded energy alignment could be formed in the ternary OSCs, which is beneficial for the facilitation of charge transfer and exciton dissociation. ${ }^{65,66}$ The work function of YF-CN was also determined to be $5.67 \mathrm{eV}$ by ultraviolet photoelectron spectroscopy (Fig. S8, ESI $\dagger$ ).

To evaluate the exciton dissociation and charge transfer in the blend, the photoluminescence (PL) spectra of PCE10, YF$\mathrm{CN}$, Y6 and their blend films were measured. As shown in Fig. S9 (ESI $\dagger$ ), the PCE10 neat film and the Y6 neat film exhibited the PL emission peak at $750 \mathrm{~nm}$ and $845 \mathrm{~nm}$, respectively. When incorporating YF-CN into the blend, the PL intensity of binary films like PCE10:YF-CN and YF-CN:Y6 was significantly quenched, suggesting the efficient exciton dissociation at both the PCE10:YF-CN and YF-CN:Y6 interfaces. ${ }^{67}$ Moreover, compared with the PCE10:Y6 film, the ternary PCE10:YF-CN:Y6 blend film displayed a greater extent of quenching approaching $100 \%$ owing to the role of YF-CN as a cascade material. These results indicate the progressive function of YF-CN for boosting the charge transfer ability in the ternary blend and the possible application for ternary OSCs with YF-CN as the third component.

To examine the photovoltaic performance of YF-CN, the OSCs were fabricated with a conventional device structure of ITO/PEDOT:PSS/active layer/PDINO/Ag. The relevant current density-voltage $(J-V)$ curves are shown in Fig. 2a and the photovoltaic performances are summarized in Table 2. Firstly, we used PCE10 as the donor and YF-CN or Y6 as the acceptor to investigate the photovoltaic properties of binary OSCs. The PCE10:Y6 (1:1.5)-based as-cast devices exhibited a PCE of $10.93 \%$, along with a high open-circuit voltage $\left(V_{\mathrm{OC}}\right)$ of $0.673 \mathrm{~V}$, a short-circuit current density $\left(J_{\mathrm{SC}}\right)$ of $25.32 \mathrm{~mA} \mathrm{~cm}^{-2}$ and a fill factor (FF) of $64.19 \%$. The PCE10:YF-CN (1:1.5)-based device annealed at $110{ }^{\circ} \mathrm{C}$ gave a rather low PCE of $1.41 \%$ but a higher $V_{\text {OC }}$ of $0.839 \mathrm{~V}$, with weaker $J_{\text {SC }}$ and $\mathrm{FF}\left(4.97 \mathrm{~mA} \mathrm{~cm}^{-2}\right.$ and $33.78 \%$, respectively). The broader absorption profile and efficient energy transfer between the donor and acceptor can work together to increase the $J_{\mathrm{SC}}$ value. The larger $V_{\mathrm{OC}}$ of the PCE10:YF-CN-based device could be ascribed to the higher LUMO level of YF-CN than that of Y6, while the lower $J_{\mathrm{SC}}$ may be due to the weaker absorption of YF-CN, combined with the PL measurement results. On the other hand, considering the high HOMO energy level of YF-CN, we also fabricated all-small-molecule OSCs based on the YF-CN : Y6 (1:1) binary active layer with thermal annealing, which provided a surprising PCE of $1.44 \%$. The above results demonstrate the efficient charge transfer at the PCE10:YF-CN and YF-CN:Y6 interface, and indicate the developmental potential of YF-CN both as a donor and an acceptor of OSCs.

Furthermore, we adjusted the photovoltaic performance of the ternary OSCs based on PCE10:Y6 with YF-CN as the third component and a constant weight ratio of $\mathrm{Y} 6$, by varying the proportion between PCE10 and YF-CN in the blend. The detailed photovoltaic results of ternary OSCs with different YF-CN contents are shown in Fig. S10 and summarized in Table S1 (ESI $\dagger$ ). It was found that the ternary as-cast OSCs with a PCE10:YF-CN:Y6 ratio of 0.8:0.2:1.5 yielded the highest
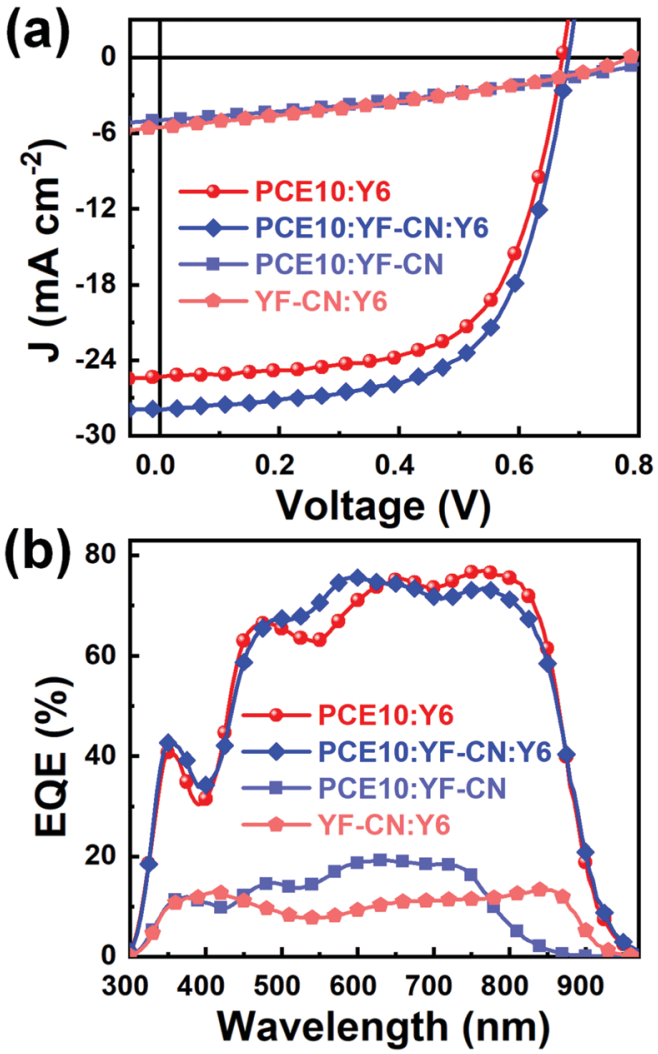

Fig. 2 (a) J-V curves and (b) EQE spectra for binary and ternary devices.

Table 2 The photovoltaic performance of the binary and ternary devices (the average values for eight devices), under the illumination of AM $1.5 \mathrm{G}$, $100 \mathrm{~mW} \mathrm{~cm}^{-2}$

\begin{tabular}{lcccl}
\hline Active layer & $V_{\mathrm{OC}}(\mathrm{V})$ & $J_{\mathrm{SC}}\left(\mathrm{mA} \mathrm{cm}^{-2}\right)$ & $\mathrm{FF}(\%)$ & PCE $(\%)$ \\
\hline PCE10:YF-CN & 0.839 & 4.97 & 33.78 & $1.41(1.32 \pm 0.09)$ \\
YF-CN:Y6 & 0.785 & 5.53 & 33.17 & $1.44(1.39 \pm 0.05)$ \\
PCE10:Y6 & 0.673 & 25.32 & 64.19 & $10.93(10.81 \pm 0.12)$ \\
PCE10:YF-CN:Y6 & 0.683 & 27.92 & 63.11 & $12.03(11.68 \pm 0.34)$
\end{tabular}

PCE of $12.03 \%$, with synergistic enhancement in $V_{\mathrm{OC}}$ and $J_{\mathrm{SC}}$ $\left(0.683 \mathrm{~V}\right.$ and $27.92 \mathrm{~mA} \mathrm{~cm}{ }^{-2}$, respectively) but a slightly lower FF of $63.11 \%$, compared with those of the above PCE10: Y6-based devices. The enhanced values of photovoltaic performances can be attributed to the terraced energy level alignment and optimized morphology of the ternary active layers. ${ }^{39}$

The external quantum efficiency (EQE) of the OSCs was measured to investigate the spectral responses of the devices (Fig. 2b). The devices based on PCE10:Y6 showed a broad photoelectrical response in the wavelength range of 300$1000 \mathrm{~nm}$. When incorporating YF-CN as the third component, the EQE values of ternary OSCs were slightly enhanced in the band between 500 and $600 \mathrm{~nm}$, which is in accordance with the enhancement of current density. For the PCE10:YF-CN, YF-CN:Y6, PCE10:Y6 and as-cast PCE10:YF-CN:Y6 based devices, the $J_{\mathrm{SC}}$ values calculated from the integration of EQE spectra were $5.11,5.75,25.31$ and $27.99 \mathrm{~mA} \mathrm{~cm}^{-2}$, respectively, 
showing good consistency with the experimental values from the $J-V$ curves within a $5 \%$ mismatch.

The space charge limited current (SCLC) test was conducted to investigate the effect of adding YF-CN on the charge transport properties of the ternary blend film. The measurement results of the electron mobilities $\left(\mu_{\mathrm{e}}\right)$ and hole mobilities $\left(\mu_{\mathrm{h}}\right)$ of the binary and ternary blend films are shown in Fig. S11 and summarized in Table S2 (ESI $\dagger$ ). The $\mu_{\mathrm{e}}$ and $\mu_{\mathrm{h}}$ values of the PCE10:Y6 blend were calculated to be $2.92 \times 10^{-4} \mathrm{~cm}^{2} \mathrm{~V}^{-1} \mathrm{~s}^{-1}$ and $3.22 \times$ $10^{-4} \mathrm{~cm}^{2} \mathrm{~V}^{-1} \mathrm{~s}^{-1}$, respectively. With $20 \mathrm{wt} \%$ YF-CN in donors, the $\mu_{\mathrm{e}}$ and $\mu_{\mathrm{h}}$ values of the PCE10:YF-CN : Y6 $(0.8: 0.2: 1.5)$ blend were increased to be $5.71 \times 10^{-4} \mathrm{~cm}^{2} \mathrm{~V}^{-1} \mathrm{~s}^{-1}$ and $3.55 \times 10^{-4} \mathrm{~cm}^{2} \mathrm{~V}^{-1} \mathrm{~s}^{-1}$, respectively. It is worth noting that the $\mu_{\mathrm{e}}$ value of the ternary active layer is almost twice that of the binary active layer. The improvement of charge mobility should be accountable for the higher $J_{\mathrm{SC}}$ values of the ternary OSCs. ${ }^{68}$

To further investigate the exciton dissociation and charge collection behavior of the devices, the relationship between photocurrent density $\left(J_{\mathrm{ph}}\right)$ and effective voltage $\left(V_{\text {eff }}\right)$ of the binary and ternary OSCs was also studied and is shown in Fig. 3a. $J_{\mathrm{ph}}$ is defined as $J_{\mathrm{ph}}=J_{\mathrm{L}}-J_{\mathrm{D}}$, where $J_{\mathrm{L}}$ and $J_{\mathrm{D}}$ are the current densities under a standard simulated light source and in the dark, respectively. $V_{\text {eff }}$ is defined as $V_{\text {eff }}=V_{0}-V_{\text {app }}$, where $V_{0}$ is the voltage at $J_{\mathrm{ph}}=0$ and $V_{\text {app }}$ is the applied voltage. The larger ratios of $J_{\mathrm{ph}} / J_{\text {sat }}$ and $J_{\text {max power }} / J_{\text {sat }}$ represent more efficient exciton dissociation and charge collection efficiency, respectively, in which $J_{\text {sat }}$ is the saturated photocurrent density and $J_{\text {max power }}$ is the maximum power output current density. ${ }^{69}$ The exciton dissociation probability $\left(P_{\text {diss }}\right)$ of the OSCs, calculated from the $J_{\mathrm{ph}} / J_{\mathrm{sat}}$ ratio under short circuit conditions, was increased from $94.0 \%$ to $96.6 \%$ by incorporating $20 \mathrm{wt} \%$ YF-CN content in donors. The charge collection probability $\left(P_{\text {coll }}\right)$ is estimated by the ratio $J_{\text {max power }} / J_{\text {sat }}$ under maximum power output conditions, which was improved from $72.0 \%$ for PCE10:Y6 device to $82.0 \%$ for PCE10:YF-CN:Y6 devices. These results demonstrate that the introduction of YF-CN helps in enhancing exciton dissociation and charge collection efficiency, contributing to a high $J_{\mathrm{SC}}$ in ternary OSCs.

To elucidate the charge recombination mechanism, light intensity $\left(P_{\text {light }}\right)$ dependence of $J_{\mathrm{SC}}$ and $V_{\mathrm{OC}}$ for binary and ternary OSCs was measured and the results are shown in Fig. $3 \mathrm{~b}$ and c, respectively. The dependence of $J_{\mathrm{SC}}$ on $P_{\text {light }}$ can be described by the power law $J_{\mathrm{SC}} \propto P_{\text {light }}^{\alpha}$, where the exponential factor $\alpha$ close to 1 reflects weak bimolecular recombination in the devices. ${ }^{70,71}$ The fitting $\alpha$ values for the binary PCE10:Y6 and ternary devices were calculated to be 0.973 and 0.979 , respectively. A larger $\alpha$ value indicates that the bimolecular recombination can be effectively decreased in the ternary OSCs. The correlation between $V_{\mathrm{OC}}$ and $P_{\text {light }}$ can be expressed as $V_{\mathrm{OC}} \propto(n k T / q) \ln \left(P_{\text {light }}\right)$, where $k$ is the Boltzmann constant, $T$ is the temperature, and $q$ is the elemental charge. In general, as the slope approaches $2 k T / q$, the trap-assisted recombination is dominant in the devices. ${ }^{72}$ The value of $n$ was 1.201 for the PCE10:Y6 device, while a smaller $n$ value of 1.198 was achieved for the ternary device, suggesting that the addition of YF-CN as the third component could also suppress the trap-assisted recombination, which is consistent with the higher $J_{\mathrm{SC}}$ of the PCE10:YF-CN:Y6 devices.

Atomic force microscopy (AFM) was conducted to probe the surface morphology of the above active layers. The AFM height images and phase images of blend films are displayed in Fig. 4. The root-mean-square (RMS) roughness values of the PCE10:Y6 and PCE10:YF-CN film were $1.25 \mathrm{~nm}$ and $1.43 \mathrm{~nm}$, respectively, which indicate a homogeneous surface in these blend films. The rougher morphology of the YF-CN:Y6 film with $16.9 \mathrm{~nm}$ may be due to the crystallinity of small molecules YF-CN and Y6. More importantly, the ternary blend film of PCE10: YF-CN:Y6 displayed a smaller RMS roughness value of $1.18 \mathrm{~nm}$ (Fig. 4d) when YF-CN was introduced into the PCE10:Y6 blend. The slight decrease of roughness suggests the good compatibility among the three materials, and the smooth morphology is conductive to the improvement of the performance of ternary OSCs. ${ }^{65}$

Aside from pursuing high efficiency, the stability of devices is another important consideration for the commercialization of organic solar cells. Thus, we studied the storage stability of the binary and ternary encapsulated devices by storing in the dark in an $\mathrm{N}_{2}$-filled glovebox at room temperature. The devices were only exposed to light irradiation during the temporary measurements. The $J-V$ curves of some devices are shown in Fig. 5 and the performance parameters are summarized in Table S3 (ESI $\dagger$ ). As shown in Fig. 5b, the ternary devices based on the PCE10:YF-CN:Y6 system encouragingly maintained $91 \%$ of its initial PCE after 360 hours of storage. In contrast, only $42 \%$ of the initial value
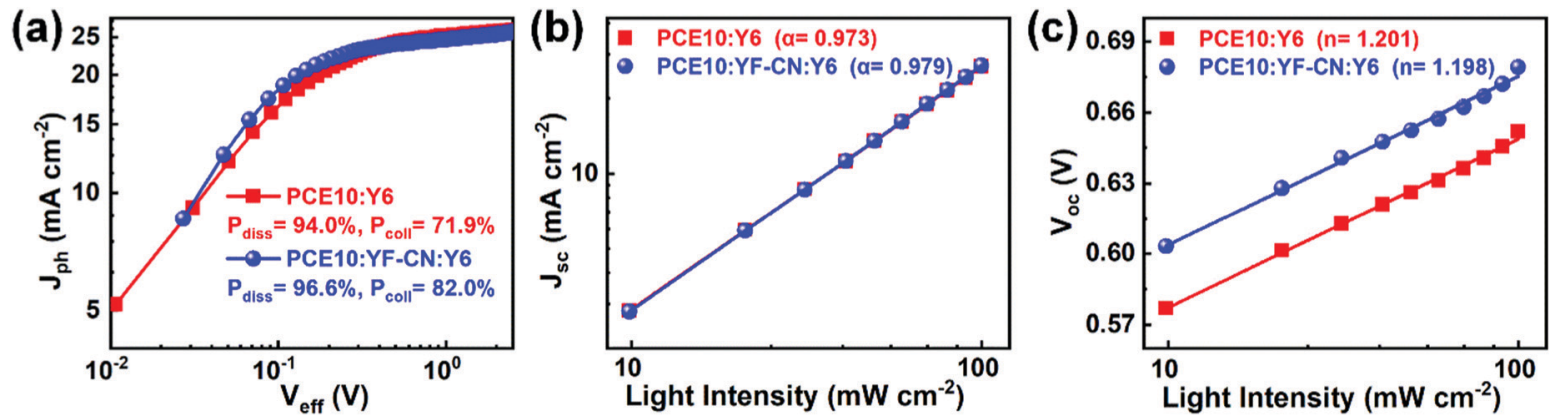

Fig. 3 (a) Photocurrent density $\left(J_{\text {ph }}\right)$ versus effective voltage $\left(V_{\text {eff }}\right)$ curves. Dependence of (b) $J_{S C}$ and (c) $V_{\text {OC }}$ on the light intensity for the binary and ternary OSCs. 
(a)

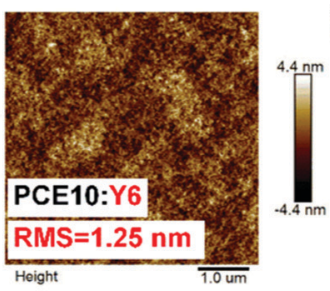

(e)

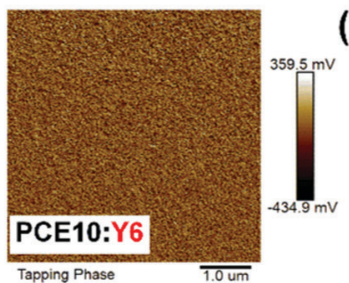

(b)

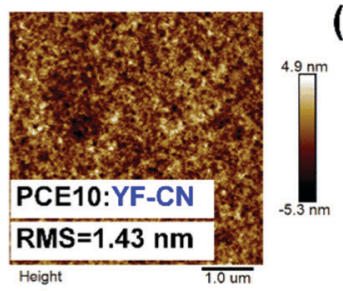

(f)

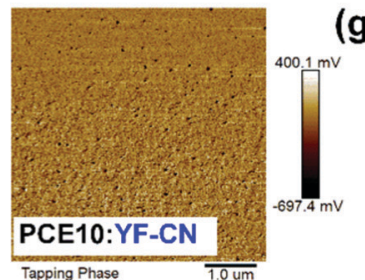

(c)

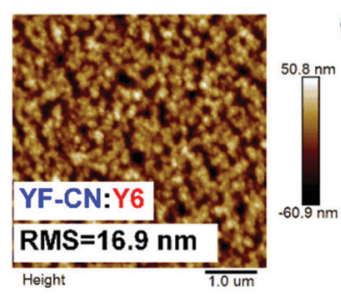

(g)

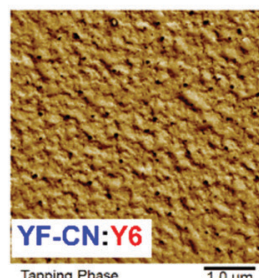

(d)

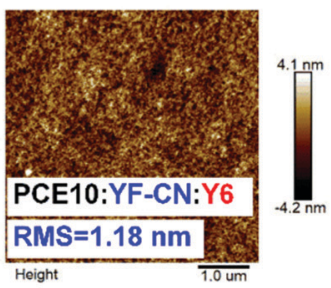

(h)

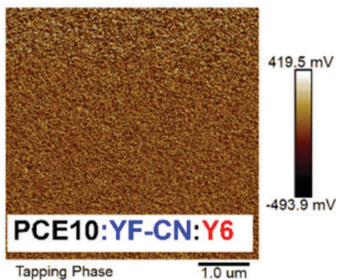

Fig. 4 The AFM height images $(a-d)$ and phase images $(e-h)$ of binary and ternary blend films.

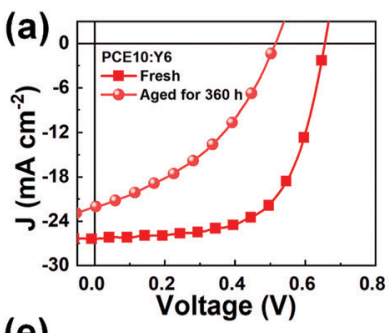

(e)

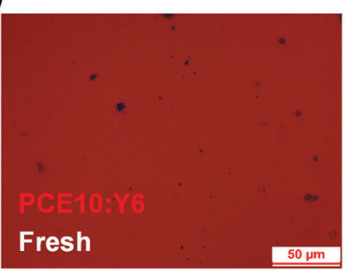

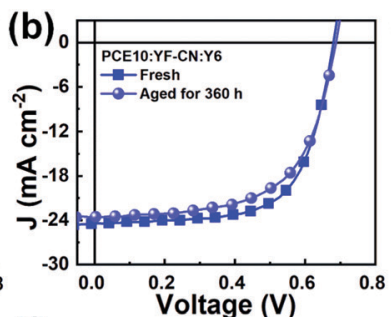

(f)

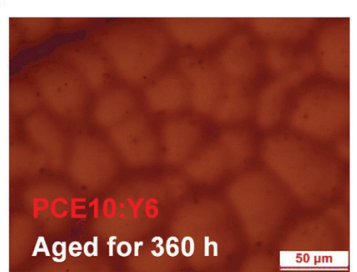

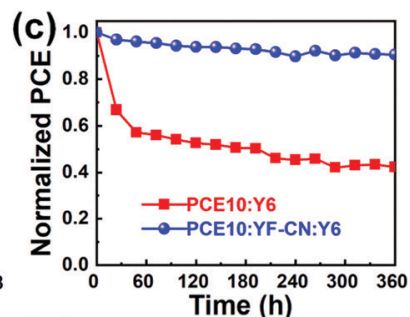

(g)

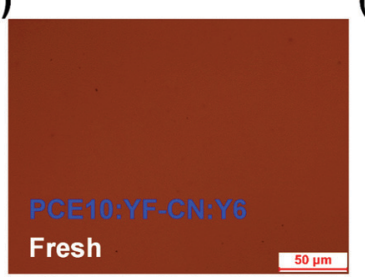

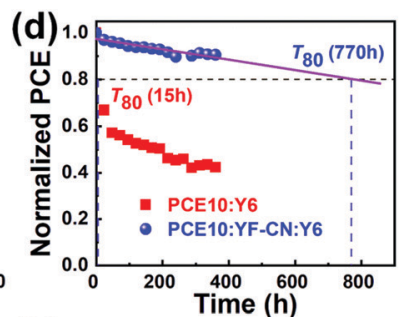

(h)

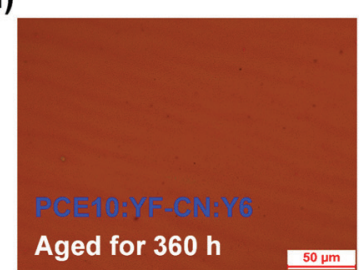

Fig. 5 The J-V curves of fresh and $360 \mathrm{~h}$ aged (a) binary and (b) ternary OSCs. (c) The storage stability of the devices at room temperature and (d) the dash line represents the $T_{80}$ lifetime analysis of the binary and ternary devices. Fluorescence microscopy images of fresh and $360 \mathrm{~h}$ aged (e and f) binary and ( $\mathrm{g}$ and $\mathrm{h}$ ) ternary OSCs.

was retained for PCE10:Y6-based binary devices under the same conditions. Furthermore, the extrapolated $T_{80}$ ( $80 \%$ of the initial PCE) lifetime analysis is shown in Fig. $5 \mathrm{c}$ and d. The extrapolated $T_{80}$ lifetime for the PCE10:YF-CN:Y6-based device is determined to be 770 hours, compared with the extremely poor $T_{80}$ lifetime of 15 hours for the PCE10:Y6-based devices. These results indicate that the addition of YF-CN could significantly improve the storage stability of ternary devices. We compare the morphology of fresh and aged binary and ternary films, as shown in Fig. 5 and Fig. S12 (ESI + ). It is interesting to note that the morphology of the binary film has changed a lot but the ternary system maintains a smooth surface, which indicates that YF-CN may work as a stabilizer by suppressing aggregation and crystallization in the ternary blend. ${ }^{73}$

\section{Conclusions}

In conclusion, an IC-free small molecule YF-CN was rationally designed and readily synthesized by Stille coupling. The robust carbon-carbon bonds of YF-CN endow it with higher intrinsic photostability than Y6. When blended with PCE10 or Y6, although the YF-CN based binary devices exhibited a PCE of about $1.4 \%$, it exhibits an unexpected bipolar charge acceptance performance, that is, it can be either used as an electron acceptor with PCE10, or as an electron acceptor with Y6, benefiting from a more suitable energy level. Moreover, when incorporating the YF-CN donor as the third component into a PCE10:Y6 system, the ternary as-cast device yielded a high PCE of $12.03 \%$, which is about $20 \%$ higher than that of the binary system. The introduction of YF-CN could form a cascaded energy level alignment and improve the compatibility as well as the miscibility of the ternary blend film. The ternary device showed improved storage stability due to the stable microstructure of the active layer. In the future, we expect to achieve high efficiency acceptors using further molecule design to properly decrease the HOMO and LUMO levels, improve the planarity between end groups and conjugated cores and increase the 
absorption range covering more sunshine radiation spectra and coefficient. More experiments on both small molecules and polymers are in progress in our lab on the above points. Different from the donors with an open-shell structure, the highly efficient acceptors have closed-shell structures. Overall, this work demonstrates a promising design concept based on dinitrile end-capped closed-shell non-quinodimethane as a stable active layer material for organic solar cells.

\section{Conflicts of interest}

There are no conflicts to declare.

\section{Acknowledgements}

The work was financially supported by the Natural Science Foundation of China $(51973063,21733005)$, the Tip-top Scientific and Technical Innovative Youth Talents of Guangdong Special Support Program (2019TQ05C890), the Ministry of Science and Technology of the People's Republic of China (2017YFA0206600), the Pearl River S\&T Nova Program of Guangzhou (201710010194) and the Fund of Guangdong Provincial Key Laboratory of Luminescence from Molecular Aggregates (2019B030301003).

\section{Notes and references}

1 J. Hou, O. Inganas, R. H. Friend and F. Gao, Nat. Mater., 2018, 17, 119-128.

2 Z. He, C. Zhong, S. Su, M. Xu, H. Wu and Y. Cao, Nat. Photonics, 2012, 6, 591-595.

3 Q. Wei, W. Liu, M. Leclerc, J. Yuan, H. Chen and Y. Zou, Sci. China: Chem., 2020, 63, 1352-1366.

4 Q. Liu, Y. Jiang, K. Jin, J. Qin, J. Xu, W. Li, J. Xiong, J. Liu, Z. Xiao, K. Sun, S. Yang, X. Zhang and L. Ding, Sci. Bull., 2020, 65, 272-275.

5 Y. Tong, Z. Xiao, X. Du, C. Zuo, Y. Li, M. Lv, Y. Yuan, C. Yi, F. Hao, Y. Hua, T. Lei, Q. Lin, K. Sun, D. Zhao, C. Duan, X. Shao, W. Li, H.-L. Yip, Z. Xiao, B. Zhang, Q. Bian, Y. Cheng, S. Liu, M. Cheng, Z. Jin, S. Yang and L. Ding, Sci. China: Chem., 2020, 63, 758-765.

6 C. Yan, S. Barlow, Z. Wang, H. Yan, A. K. Y. Jen, S. R. Marder and X. Zhan, Nat. Rev. Mater., 2018, 3, 18003.

7 J. Lee, S. M. Lee, S. Chen, T. Kumari, S. H. Kang, Y. Cho and C. Yang, Adv. Mater., 2019, 31, 1804762.

8 Y. Lin, J. Wang, Z. G. Zhang, H. Bai, Y. Li, D. Zhu and X. Zhan, Adv. Mater., 2015, 27, 1170-1174.

9 W. Chen and Q. Zhang, J. Mater. Chem. C, 2017, 5, 1275-1302.

10 J. Yuan, Y. Zhang, L. Zhou, G. Zhang, H.-L. Yip, T.-K. Lau, X. Lu, C. Zhu, H. Peng, P. A. Johnson, M. Leclerc, Y. Cao, J. Ulanski, Y. Li and Y. Zou, Joule, 2019, 3, 1140-1151.

11 S. Liu, J. Yuan, W. Deng, M. Luo, Y. Xie, Q. Liang, Y. Zou, Z. He, H. Wu and Y. Cao, Nat. Photonics, 2020, 14, 300-305.
12 Y. Cui, H. Yao, J. Zhang, K. Xian, T. Zhang, L. Hong, Y. Wang, Y. Xu, K. Ma, C. An, C. He, Z. Wei, F. Gao and J. Hou, Adv. Mater., 2020, 32, 1908205.

13 X. Wan, C. Li, M. Zhang and Y. Chen, Chem. Soc. Rev., 2020, 49, 2828-2842.

14 Z. Luo, R. Ma, T. Liu, J. Yu, Y. Xiao, R. Sun, G. Xie, J. Yuan, Y. Chen, K. Chen, G. Chai, H. Sun, J. Min, J. Zhang, Y. Zou, C. Yang, X. Lu, F. Gao and H. Yan, Joule, 2020, 4, 1236-1247.

15 Y. Ma, M. Zhang, S. Wan, P. Yin, P. Wang, D. Cai, F. Liu and Q. Zheng, Joule, 2021, 5, 197-209.

16 C. Li, J. Zhou, J. Song, J. Xu, H. Zhang, X. Zhang, J. Guo, L. Zhu, D. Wei, G. Han, J. Min, Y. Zhang, Z. Xie, Y. Yi, H. Yan, F. Gao, F. Liu and Y. Sun, Nat. Energy, 2021, 6, 605-613.

17 S. Chen, L. Feng, T. Jia, J. Jing, Z. Hu, K. Zhang and F. Huang, Sci. China: Chem., 2021, 64, 1192-1199.

18 Z. Luo, R. Sun, C. Zhong, T. Liu, G. Zhang, Y. Zou, X. Jiao, J. Min and C. Yang, Sci. China: Chem., 2020, 63, 361-369.

19 P. Bi and X. Hao, Sol. RRL, 2019, 3, 1800263.

20 D. Zhou, W. You, H. Xu, Y. Tong, B. Hu, Y. Xie and L. Chen, J. Mater. Chem. A, 2020, 8, 23096-23122.

21 Q. An, J. Wang, W. Gao, X. Ma, Z. Hu, J. Gao, C. Xu, M. Hao, X. Zhang, C. Yang and F. Zhang, Sci. Bull., 2020, 65, 538-545. 22 Y. Chang, J. Zhang, Y. Chen, G. Chai, X. Xu, L. Yu, R. Ma, H. Yu, T. Liu, P. Liu, Q. Peng and H. Yan, Adv. Energy Mater., 2021, 11, 2100079.

23 M. Jiang, H. Bai, H. Zhi, L. Yan, H. Y. Woo, L. Tong, J. Wang, F. Zhang and Q. An, Energy Environ. Sci., 2021, 14, 3945-3953.

24 Y. Zeng, D. Li, Z. Xiao, H. Wu, Z. Chen, T. Hao, S. Xiong, Z. Ma, H. Zhu, L. Ding and Q. Bao, Adv. Energy Mater., 2021, 11, 2101338.

25 M. Zhang, L. Zhu, T. Hao, G. Zhou, C. Qiu, Z. Zhao, N. Hartmann, B. Xiao, Y. Zou, W. Feng, H. Zhu, M. Zhang, Y. Zhang, Y. Li, T. P. Russell and F. Liu, Adv. Mater., 2021, 33, 2007177.

26 R. Ma, Y. Tao, Y. Chen, T. Liu, Z. Luo, Y. Guo, Y. Xiao, J. Fang, G. Zhang, X. Li, X. Guo, Y. Yi, M. Zhang, X. Lu, Y. Li and H. Yan, Sci. China: Chem., 2021, 64, 581-589.

27 J. Gao, J. Wang, Q. An, X. Ma, Z. Hu, C. Xu, X. Zhang and F. Zhang, Sci. China: Chem., 2019, 63, 83-91.

28 Z. Su, Z. Zhang, G. Xie, Y. Zhang, X. Zhang, W. Zhang and J. Zhang, Dyes Pigm., 2021, 192, 109434.

29 M. Zhang, L. Zhu, G. Zhou, T. Hao, C. Qiu, Z. Zhao, Q. Hu, B. W. Larson, H. Zhu, Z. Ma, Z. Tang, W. Feng, Y. Zhang, T. P. Russell and F. Liu, Nat. Commun., 2021, 12, 309.

30 T. Liu, T. Yang, R. Ma, L. Zhan, Z. Luo, G. Zhang, Y. Li, K. Gao, Y. Xiao, J. Yu, X. Zou, H. Sun, M. Zhang, T. A. Dela Peña, Z. Xing, H. Liu, X. Li, G. Li, J. Huang, C. Duan, K. S. Wong, X. Lu, X. Guo, F. Gao, H. Chen, F. Huang, Y. Li, Y. Li, Y. Cao, B. Tang and H. Yan, Joule, 2021, 5, 914-930.

31 J. Qin, Z. Chen, P. Bi, Y. Yang, J. Zhang, Z. Huang, Z. Wei, C. An, H. Yao, X. Hao, T. Zhang, Y. Cui, L. Hong, C. Liu, Y. Zu, C. He and J. Hou, Energy Environ. Sci., 2021, 14, 5903-5910. 
32 L. Xu, W. Tao, H. Liu, J. Ning, M. Huang, B. Zhao, X. Lu and S. Tan, J. Mater. Chem. A, 2021, 9, 11734-11740.

33 X. Xu, Y. Li and Q. Peng, Adv. Mater., 2021, 2107476.

34 Y. Yin, L. Zhan, M. Liu, C. Yang, F. Guo, Y. Liu, S. Gao, L. Zhao, H. Chen and Y. Zhang, Nano Energy, 2021, 90, 106538.

35 C. Zhao, J. Wang, X. Zhao, Z. Du, R. Yang and J. Tang, Nanoscale, 2021, 13, 2181-2208.

36 Z. Chen, W. Song, K. Yu, J. Ge, J. Zhang, L. Xie, R. Peng and Z. Ge, Joule, 2021, 5, 2395-2407.

37 T. Zhang, C. An, P. Bi, Q. Lv, J. Qin, L. Hong, Y. Cui, S. Zhang and J. Hou, Adv. Energy Mater., 2021, 2101705.

38 Y. Cai, Y. Li, R. Wang, H. Wu, Z. Chen, J. Zhang, Z. Ma, X. Hao, Y. Zhao, C. Zhang, F. Huang and Y. Sun, Adv. Mater., 2021, 33, 2101733.

39 F. Liu, L. Zhou, W. Liu, Z. Zhou, Q. Yue, W. Zheng, R. Sun, W. Liu, S. Xu, H. Fan, L. Feng, Y. Yi, W. Zhang and X. Zhu, Adv. Mater., 2021, 33, 2100830.

40 P. Bi, S. Zhang, Z. Chen, Y. Xu, Y. Cui, T. Zhang, J. Ren, J. Qin, L. Hong, X. Hao and J. Hou, Joule, 2021, 5, 2408-2419.

41 Y. Cui, Y. Xu, H. Yao, P. Bi, L. Hong, J. Zhang, Y. Zu, T. Zhang, J. Qin, J. Ren, Z. Chen, C. He, X. Hao, Z. Wei and J. Hou, Adv. Mater., 2021, 33, 2102420.

42 K. Yu, W. Song, Y. Li, Z. Chen, J. Ge, D. Yang, J. Zhang, L. Xie, C. Liu and Z. Ge, Small Struct., 2021, 2, 2100099.

43 O. R. Yamilova, I. V. Martynov, A. S. Brandvold, I. V. Klimovich, A. H. Balzer, A. V. Akkuratov, I. E. Kusnetsov, N. Stingelin and P. A. Troshin, Adv. Energy Mater., 2020, 10, 1903163.

44 X. Du, T. Heumueller, W. Gruber, O. Almora, A. Classen, J. Qu, F. He, T. Unruh, N. Li and C. J. Brabec, Adv. Mater., 2020, 32, 1908305.

45 Y. Li, T. Li and Y. Lin, Mater. Chem. Front., 2021, 5, 2907-2930.

46 Y. Zou and L. Ye, Chem, 2021, 7, 2853-2854.

47 L. Ciammaruchi, O. Zapata-Arteaga, E. Gutiérrez-Fernández, J. Martin and M. Campoy-Quiles, Mater. Adv., 2020, 1, 2846-2861.

48 W. Li, D. Liu and T. Wang, Adv. Funct. Mater., 2021, 31, 2104552.

49 Z. X. Liu, Z. P. Yu, Z. Shen, C. He, T. K. Lau, Z. Chen, H. Zhu, X. Lu, Z. Xie, H. Chen and C. Z. Li, Nat. Commun., 2021, 12, 3049.

50 Y. Che, M. R. Niazi, R. Izquierdo and D. F. Perepichka, Angew. Chem., Int. Ed., 2021, 60, 24833-24837.

51 H. Liu, W. Wang, Y. Zhou and Z. A. Li, J. Mater. Chem. A, 2021, 9, 1080-1088.

52 X. Zhu, S. Liu, Q. Yue, W. Liu, S. Sun and S. Xu, CCS Chem., 2021, 3, 1070-1080.
53 Q.-Q. Zhang, Y. Li, D. Wang, Z. Chen, Y. Li, S. Li, H. Zhu, X. Lu, H. Chen and C.-Z. Li, Bull. Chem. Soc. Jpn., 2021, 94, 183-190.

54 H. Sun, X. Song, J. Xie, P. Sun, P. Gu, C. Liu, F. Chen, Q. Zhang, Z. K. Chen and W. Huang, ACS Appl. Mater. Interfaces, 2017, 9, 29924-29931.

55 Y. Li, L. Li, Y. Wu and Y. Li, J. Phys. Chem. C, 2017, 121, 8579-8588.

56 Z. Chen, W. Li, M. A. Sabuj, Y. Li, W. Zhu, M. Zeng, C. S. Sarap, M. M. Huda, X. Qiao, X. Peng, D. Ma, Y. Ma, N. Rai and F. Huang, Nat. Commun., 2021, 12, 5889.

57 Z. Chen, W. Li, Y. Zhang, Z. Wang, W. Zhu, M. Zeng and Y. Li, J. Phys. Chem. Lett., 2021, 12, 9783-9790.

58 Z. X. Chen, Y. Li and F. Huang, Chem, 2021, 7, 288-332.

59 Y.-J. Cheng, S.-H. Yang and C.-S. Hsu, Chem. Rev., 2009, 109, 5868-5923.

60 C. Sun, S. Qin, R. Wang, S. Chen, F. Pan, B. Qiu, Z. Shang, L. Meng, C. Zhang, M. Xiao, C. Yang and Y. Li, J. Am. Chem. Soc., 2020, 142, 1465-1474.

61 H. Yao, Y. Cui, D. Qian, C. S. Ponseca, Jr., A. Honarfar, Y. Xu, J. Xin, Z. Chen, L. Hong, B. Gao, R. Yu, Y. Zu, W. Ma, P. Chabera, T. Pullerits, A. Yartsev, F. Gao and J. Hou, J. Am. Chem. Soc., 2019, 141, 7743-7750.

62 H. Yao, D. Qian, H. Zhang, Y. Qin, B. Xu, Y. Cui, R. Yu, F. Gao and J. Hou, Chin. J. Chem., 2018, 36, 491-494.

63 F. Cai, H. Peng, H. Chen, J. Yuan, J. Hai, T.-K. Lau, J. Wang, Y. Hu, W. Liu, X. Lu and Y. Zou, J. Mater. Chem. A, 2020, 8, 15984-15991.

64 C. Cao, H. Lai, H. Chen, Y. Zhu, M. Pu, N. Zheng and F. He, J. Mater. Chem. A, 2021, 9, 16418.

65 W. Jiang, R. Yu, Z. Liu, R. Peng, D. Mi, L. Hong, Q. Wei, J. Hou, Y. Kuang and Z. Ge, Adv. Mater., 2018, 30, 1703005.

66 Z. Liu and H. Wang, Dyes Pigm., 2021, 192, 109424.

67 B.-H. Jiang, C.-P. Chen, H.-T. Liang, R.-J. Jeng, W.-C. Chien and Y.-Y. Yu, Dyes Pigm., 2020, 181, 108613.

68 Q. Ma, Z. Jia, L. Meng, J. Zhang, H. Zhang, W. Huang, J. Yuan, F. Gao, Y. Wan, Z. Zhang and Y. Li, Nano Energy, 2020, 78, 105272.

69 H. Tang, H. Chen, C. Yan, J. Huang, P. W. K. Fong, J. Lv, D. Hu, R. Singh, M. Kumar, Z. Xiao, Z. Kan, S. Lu and G. Li, Adv. Energy Mater., 2020, 10, 2001076.

70 W. Chen, G. Huang, X. Li, H. Wang, Y. Li, H. Jiang, N. Zheng and R. Yang, ACS Appl. Mater., 2018, 10, 42747-42755.

71 T. Fukuhara, Y. Tamai and H. Ohkita, Sustainable Energy Fuels, 2020, 4, 4321-4351.

72 S. Song, K. T. Lee, C. W. Koh, H. Shin, M. Gao, H. Y. Woo, D. Vak and J. Y. Kim, Energy Environ. Sci., 2018, 11, 3248-3255.

73 Y. Qin, N. Balar, Z. Peng, A. Gadisa, I. Angunawela, A. Bagui, S. Kashani, J. Hou and H. Ade, Joule, 2021, 5, 2129-2147. 